\title{
Asymptomatic carotid stenosis
}

\section{Medicine alone or combined with carotid revascularization}

Donald V. Heck, MD

Gary S. Roubin, MD,

$\mathrm{PhD}$

Kenneth G. Rosenfield, MD, MHCDS

William A. Gray, MD

Christopher J. White, MD

Tudor G. Jovin, MD

Jon S. Matsumura, MD

Brajesh K. Lal, MD

Barry T. Katzen, MD

Guilherme Dabus, MD

Brian T. Jankowitz, MD

Thomas G. Brott, MD

Correspondence to

Dr. Heck:

dvheck66@gmail.com

Editorials, pages 1986, 1988 , and 1990

\section{ABSTRACT}

Two positive randomized trials established carotid endarterectomy (CEA) as a superior treatment to medical management alone for the treatment of asymptomatic carotid artery stenosis. However, advances in medical therapy have led to an active and spirited debate about the best treatment for asymptomatic carotid stenosis. The Carotid Revascularization and Medical Management for Asymptomatic Carotid Stenosis (CREST 2) trial aims to better define the best treatment for the average patient with severe asymptomatic carotid stenosis. Enrollment in the trial may be hampered by strong opinions on either side of the debate. It is important to realize that equipoise exists and that neither the old data on CEA nor the new data on optimal medical therapy provide a rigorous answer. The assumption that medical therapy has already been proven superior to revascularization procedures may hinder both enrollment in the trial and technical advancements in revascularization procedures. Neurology ${ }^{\circledR}$ 2017;88:2061-2065

\section{GLOSSARY}

ACAS = Asymptomatic Carotid Atherosclerosis Study; $\mathbf{A C S T}=$ Asymptomatic Carotid Surgery Trial; $\mathbf{A C T} 1$ = Asymptomatic Carotid Stenosis Stenting vs Endarterectomy Trial; C2R = Carotid Revascularization and Medical Management for Asymptomatic Carotid Stenosis Registry; CAS = carotid stenting; CEA = carotid endarterectomy; CMS = Centers for Medicare Services; CREST = Carotid Revascularization Endarterectomy versus Stenting Trial; CREST $\mathbf{2}=$ Carotid Revascularization and Medical Management for Asymptomatic Carotid Stenosis; FDA = Food and Drug Administration; IMC = Interventional Management Committee; SAMMPRIS = Stenting and Aggressive Medical Management for Preventing Recurrent Stroke in Intracranial Stenosis trial.

Recently, Drs. Spence and Naylor ${ }^{1}$ called for cessation of all carotid revascularization, carotid endarterectomy (CEA) and carotid stenting (CAS), of low-risk asymptomatic carotid stenosis outside of the Carotid Revascularization and Medical Management for Asymptomatic Carotid Stenosis (CREST 2) randomized trial. In 2 trials separated by a decade, CEA has twice been shown superior to nonoperative management of asymptomatic carotid stenosis, with an absolute risk reduction of $5 \%-6 \%$ over 5 years. ${ }^{2,3}$ Neither trial employed what anyone would consider optimal medical management today, and hence Drs. Spence and Naylor have declared them obsolete. CAS has now been shown in 2 separate randomized trials to be a lowrisk procedure with comparable periprocedural morbidity to CEA using a composite endpoint of stroke, death, and myocardial infarction, with equivalent long-term stroke prevention. ${ }^{4-6}$ Drs. Spence and Naylor correctly point out that, while both of these landmark trials demonstrated some of the lowest perioperative event rates for both CEA and CAS to date, they did not answer the question of whether to revascularize the patient with severe asymptomatic carotid stenosis in addition to providing the best medical treatment. Substantial treatment pattern discrepancies continue to exist among the United States, Canada, and Europe, with revascularization much more likely to be offered to asymptomatic patients in the United States. ${ }^{7}$ Those of us working diligently to randomize patients in CREST 2 agree there is equipoise and do not presume to know the answer.

Carotid stenosis provokes anxiety in both patients and their primary care physicians, and with reason. Between $13 \%$ and $32 \%$ of acute intracranial large vessel occlusions are caused by cervical atherosclerotic carotid stenosis or occlusion. ${ }^{8-11}$ Patients and their primary physicians understandably seek expert consultation and treatment when a diagnosis of carotid stenosis is made. Indeed there is compelling evidence that optimal medical management can have a dramatic effect in secondary stroke prevention. In the Stenting and Aggressive Medical

From the Novant Health Forsyth Comprehensive Stroke Center (D.V.H.), Winston Salem, NC; Cardiovascular Associates of the Southeast (G.S.R.), Birmingham, AL; Massachusetts General Hospital (K.G.R.), Boston; Main Line Health System/Lankenau Heart Institute (W.A.G.), Wynnewood, PA; Ochsner Medical Institutions (C.J.W.), New Orleans, LA; University of Pittsburgh Medical Center (T.G.J., B.T.J.), PA; University of Wisconsin School of Medicine and Public Health (J.S.M.), Madison; University of Maryland Medical Center (B.K.L.), Baltimore, MD; Miami Cardiac and Vascular Institute (B.T.K., G.D.); and Mayo Clinic (T.G.B.), Jacksonville, FL.

Go to Neurology.org for full disclosures. Funding information and disclosures deemed relevant by the authors, if any, are provided at the end of the article. 
Management for Preventing Recurrent Stroke in Intracranial Stenosis trial (SAMMPRIS), the incidence of recurrent stroke was decreased by half for patients with symptomatic intracranial atherosclerosis compared to the rate in the Warfarin vs Aspirin for Symptomatic Intracranial Disease (WASID) trial. SAMMPRIS used a strategy of optimal control of hypertension, dyslipidemia, and diabetes, with antiplatelet therapy and lifestyle coaching to encourage smoking cessation and exercise. ${ }^{12}$ In addition, there is evidence that the annual incidence of stroke with optimal medical management of asymptomatic carotid stenosis may be lower than the 2\%/year observed in both the Asymptomatic Carotid Atherosclerosis Study (ACAS) and the Asymptomatic Carotid Surgery Trial (ACST). ${ }^{13-15}$ Drs. Spence and Naylor note that evidence suggests that the rate may in fact be as low as $0.5 \%-1 \%$ /year, which is similar to the annual rate after successful CEA or stenting. It is important to note that the latter point is not proven in a prospective randomized trial with rigorous follow-up and independent adjudication of outcomes. But, at a minimum, there appears to be equipoise. We are equally uncertain which answer is correct (or incorrect). Hence it is premature, and certainly without scientific basis, to declare ACAS and ACST obsolete, and to suggest that no low-risk asymptomatic patient should have a carotid revascularization procedure (CEA or CAS) outside of a trial. While such a policy would indeed facilitate earlier completion of randomized trials, many patients choose not to or cannot participate in randomized trials for a variety of reasons. Given the data from ACAS and ACST and the current equipoise, it seems unreasonable that a patient with low-risk severe asymptomatic stenosis should never be offered any revascularization procedure, CEA or CAS, outside of a trial. One should note that there are many potential ways to identify the clinically asymptomatic patient who is low risk for embolism, including transcranial Doppler and high-resolution MRI plaque imaging, but these methods themselves are unproven in a randomized trial, and are not universally available. Consider the reverse situation, that medical management had twice been shown superior to CEA for asymptomatic carotid stenosis, and new data now suggest a lower incidence of stroke with improved CEA and stenting. Would anyone be calling for the suspension of medical management alone without a randomized trial? We need evidence, not eminence, on either side of the debate.

Unlike medical treatments, surgical procedures require the coming together of training, procedural experience, and technology. This is especially true for endovascular procedures. The original trials that led to the Food and Drug Administration (FDA) approval of the Merci retriever and the Penumbra system for the treatment of large vessel occlusion stroke were not designed to prove superiority of the procedure to the natural history of the disease. ${ }^{16,17}$ Despite the lack of evidence of clinical benefit, the FDA approval of the devices was a seminal event in the field of endovascular stroke intervention. The Centers for Medicare Services (CMS) followed with reimbursement to hospitals and physicians for the procedure, allowing physicians to obtain procedural experience, and providing an impetus for industry to invest in new and better devices. In 2013, 3 trials were completed, all failing to demonstrate the clinical efficacy of endovascular stroke treatment. ${ }^{18-20}$ The results led to similar calls for the cessation of endovascular stroke treatment outside of trials. ${ }^{21}$ A quantum leap in the technology, the development of stent retrievers, had already occurred. ${ }^{22,23}$ Contemporaneous improvements in aspiration catheter technology were also occurring, along with a better understanding of the populations most likely to benefit. Within 2 years, no fewer than 5 randomized trials were completed, establishing mechanical embolectomy as, by far, the most important surgical intervention ever for ischemic stroke. ${ }^{9-11,24,25}$ Where would we be had we suspended all endovascular stroke treatment until clinical benefit had been unequivocally proven? The effect would have been an abrupt decrease in the number of physicians with the necessary procedural training and experience to perform the procedures. Industry investment in new technology would have dwindled, which would have left us nowhere close to where we are today in our ability to help patients with acute stroke.

The risk of stroke with CEA performed by experienced surgeons in asymptomatic patients is at an all-time low: $1.4 \%$ in the Carotid Revascularization Endarterectomy versus StentingTrial (CREST) and $1.7 \%$ in the Asymptomatic Carotid Stenosis Stenting vs Endarterectomy Trial (ACT 1). CAS can be performed, by experienced interventionists and with proper patient selection, with results equivalent to CEA, and with no difference in cost. ${ }^{26,27}$ Comparable long-term postprocedural health-related quality of life has been documented for both CAS and CEA, with advantages of CAS in the first month. ${ }^{28}$ It is undeniable, however, that the incidence of nondisabling periprocedural stroke with CAS has been significantly higher or numerically slightly higher than CEA in every randomized trial comparing the 2 techniques. The 30-day incidence of stroke was $1.3 \%$ higher in ACT 1 and $1.1 \%$ higher in CREST for asymptomatic patients, and $2.1 \%$ higher overall for the CAS patients in CREST. 5,6,29 Although only a surrogate for clinical outcomes, clinically silent ischemic lesions on MRI diffusion imaging may also be more frequent 
with CAS. ${ }^{30}$ Considering that patients given 2 equally efficacious procedures generally prefer a less invasive treatment, why must we accept these circumstances as unchangeable?

The limited scope of reimbursement for CAS in the United States has resulted in an insubstantial rate of technology development. While new devices for the treatment of large-vessel occlusion stroke with mechanical embolectomy come at a dizzying pace, carotid stent technology has not changed substantially in a decade. Development of new and improved embolic protection systems, and meaningful trials to compare different strategies, have been equally deficient. There is one new stent design being tested in a clinical trial, and another soon to be. There is one new FDA-approved flow-reversal strategy, and others that have been withdrawn because of the absence of a market, given the absence of CMS coverage for CAS for most symptomatic and all asymptomatic patients. Simultaneously, physician experience with CAS is declining dramatically since 2014 due to the closure of all CMS-approved registries. These registries had permitted treatment and reimbursement for symptomatic patients with 50\%-69\% stenosis and asymptomatic patients with $80 \%-99 \%$ stenosis at higher than usual risk for CEA (treatment of highrisk symptomatic patients with 70\%-99\% stenosis had already been approved by CMS). As the Interventional Management Committee (IMC) of CREST 2 reviewed applicants for the carotid stent arm of the trial, rarely had an applicant performed the initial qualification goal of 25 or more carotid stent procedures within the previous year, and most had done substantially fewer.

The lack of investment in new carotid stent technologies and trials to assess them, combined with declining physician expertise, is not a recipe for success. The advances that have been achieved with improved medical management must be paralleled with complementary advances in interventional treatments. A moratorium on all carotid revascularization procedures outside of randomized trials will only exacerbate the situation, instead of fostering an environment where the procedure can fulfill its promise through proper physician training and improved technology. Drs. Spence and Naylor fear overuse of CAS on inappropriate patients. As with all surgical and procedural treatments, we must find with CAS (and endarterectomy) the proper balance of the right procedures performed by the right physicians. Surely that balance lies somewhere among an explosion of unnecessary procedures, unqualified physicians performing procedures 2 times a year, and a moratorium on all procedures.

The CREST 2 Registry (C2R) was designed to provide an avenue for potential carotid stent physicians within the trial to enhance and maintain experience with the procedure in order to ensure the best possible outcomes in the stent arm of the trial. Physicians are vetted by the IMC after review of procedural records from 25 consecutive cases, and are required to enroll all future patients in 1 of 2 national registries, with the data then transferred to the $\mathrm{C} 2 \mathrm{R}$. All physicians undergo training on patient selection. Once an investigator is approved for the randomized phase of the trial, CREST 2 eligible patients can only be entered in the registry if they refuse participation in the randomized trial, and even then a site may not enter more eligible patients in the registry than in the randomized trial. Hence there is far more quality oversight for CAS than for CEA, or for that matter, the vast majority of surgical procedures. Leaders in the CAS community are, and always have been, strong advocates of outcomes tracking, and we agree that both CAS and CEA should be performed at centers that track results and demonstrate quality outcomes.

Reimbursement for CAS in asymptomatic patients who are Medicare beneficiaries is linked to participation in the CREST 2 companion registry or the randomized trial, which promotes trial enrollment. A similar situation does not exist for CEA. Linking reimbursement for CEA in asymptomatic patients to participation in a companion registry or the CREST 2 trial would undoubtedly also enhance enrollment, but may be controversial in limiting access to a procedure that has the weight of 2 randomized trials behind it. One additional barrier to trial enrollment is the practice pattern in the United States. Neurologists are infrequently involved in the management of asymptomatic carotid stenosis, often bypassed from the primary care physician to the surgeon or interventionalist. Perhaps neurologists interested in vascular disease should play a more active role in the medical management and triage of these patients.

Drs. Spence and Naylor also question the generalizability of the surgical results in CREST and ACT 1 to the community at large. They cite reviews of administrative datasets indicating higher than acceptable complication rates for CAS, and question the relevance of these trials in making treatment decisions in the asymptomatic patient. ${ }^{31}$ First, reimbursement policy in the United States has remarkably biased the performance of stenting. CAS has been utilized in a cohort ;of patients who are elderly or, as defined by the CMS, have significant comorbidities. We agree that this group of patients requires additional study. They were not the subject of CREST or ACT 1. Second, these trials included hundreds of surgeons and interventionalists from over 100 medical centers in North America and 
should reflect what is possible and can reasonably be expected with appropriate training. Questions of generalizability will always exist, whether the subject is surgery, or the intensively managed optimized medical treatment in SAMMPRIS and CREST 2. The medical treatment protocols in those trials may also be difficult to reproduce in the community at large for a variety of reasons, including access to care, physician expertise, and patient compliance due to social or cost barriers.

The results of CREST 2 should provide great insight into the best management of asymptomatic carotid stenosis. However, completing the trial requires recognition on both the medical and surgical sides of the debate that we do not know the answer to the question "How do we best manage the average asymptomatic carotid stenosis patient?" As a community, we must suspend our biases and attempt to enroll every eligible patient. However, even if the trial shows equivalence of optimal medical therapy and optimal medical therapy plus revascularization, it is certain that there will be a continued need for carotid revascularization procedures (CEA and CAS) in selected subgroups of both symptomatic and asymptomatic patients. Further, CEA, CAS, and medical therapy will never be equivalent for all patients. There are clearly patient characteristics that may favor one surgical treatment over another, or no procedure at all. Carotid stenosis patients should be cared for by an individual, or team of individuals working together, with the expertise to offer all treatment options. We must foster an environment that aims to do the right thing for the right patient, and also one that assures adequate physician training to perform procedures and enables advances in technology to make those procedures as safe as possible.

\section{AUTHOR CONTRIBUTIONS}

Dr. Heck is the primary author, responsible for the initial manuscript and incorporation of the content contributed by the other authors, and the final edits and content of the commentary. Dr. Roubin provided content and critical review of the manuscript. Dr. Rosenfield provided content and critical review of the manuscript. Dr. Gray provided content and critical review of the manuscript. Dr. White provided critical review and substantive editorial input. Dr. Jovin provided critical review and substantive editorial input. Dr. Matsumura provided critical review and substantive editorial input. Dr. Lal provided critical review and substantive editorial input. Dr. Katzen provided critical review and substantive editorial input. Dr. Dabus provided critical review and substantive editorial input. Dr. Jankowitz provided critical review and substantive editorial input. Dr. Brott provided content and critical review of the manuscript.

\section{STUDY FUNDING}

This manuscript is funded in part by NIH CREST 2 U01 NS080168 03

\section{DISCLOSURE}

D. Heck is a consultant for Stryker and has served as an investigator for WL Gore. G. Roubin is a consultant for Cook Medical. K. Rosenfield serves on the advisory board for the following companies: Silk Road, Cardinal Health, and Abbott, and has an equity interest in Contego.
W. Gray is a consultant and receives institutional research support from WL Gore. C. White reports no disclosures relevant to the manuscript. T. Jovin is a consultant for Codman Neurovascular, and has an equity interest in Blockade Medical, Silk Road, Anaconda, and Remedy Pharmaceuticals, and serves as an unpaid member of Medtronic Advisory Board. J. Matsumura has grant support from Abbott, WL Gore, Cook, Medtronic, and Endologics. B. Lal reports no disclosures relevant to the manuscript. B. Katzen serves on the advisory boards of Boston Scientific, Medtronic Vascular, WL Gore, and Philips Healthcare. G. Dabus is a consultant for Medtronic, Microvention, and Penumbra, and has an equity interest in Medina, Surpass, and InNeuroCo. B. Jankowitz and T. Brott report no disclosures relevant to the manuscript. Go to Neurology.org for full disclosures.

Received September 1, 2016. Accepted in final form February 13, 2017.

\section{REFERENCES}

1. Spence D, Naylor AR. Embolectomy, stenting, or neither for asymptomatic carotid-artery stenosis. N Engl J Med 2016;374:1087-1088.

2. Anonymous. Endarterectomy for asymptomatic carotid artery stenosis: executive committee for the Asymptomatic Carotid Atherosclerosis Study. JAMA 1995;273: 1421-1428.

3. MRC Asymptomatic Carotid Surgery Trial (ACST) Collaborative Group. Prevention of disabling and fatal strokes by successful carotid endarterectomy in patients without recent neurological symptoms: a randomized controlled trial. Lancet 2004;363:1491-1502.

4. Brott TG, Hobson RW, Howard G, et al. Stenting versus endarterectomy for treatment of carotid artery stenosis. N Engl J Med 2010;363:11-23.

5. Brott TG, Howard G, Roubin GS, et al. Long-term results of stenting versus endarterectomy for carotid artery stenosis. N Engl J Med 2016;374:1021-1031.

6. Rosenfeld K, Matsumura JS, Chaturvedi S, et al. Randomized trial of stent versus surgery for asymptomatic carotid stenosis. N Engl J Med 2016;374:1011-1020.

7. Vikatmaa P, Mitchell D, Jensen LP, et al. Variation in clinical practice in carotid surgery in nine countries 2005-2010. Eur J Vasc Endovasc Surg 2012;44:11-17.

8. Powers WJ, Derdeyn CP, Biller J, et al. 2015 AHA/ASA focused update of the 2013 guidelines for the early management of patients with acute ischemic stroke. Stroke 2015;46:3020-3035.

9. Berkhemer OA, Eransen PS, Beumer D, et al. A randomized trial of intraarterial treatment of acute ischemic stroke. N Engl J Med 2015;372:11-20.

10. Goyal M, Demchuk AM, Menon BK, et al. Randomized assessment of rapid endovascular treatment of ischemic stroke. N Engl J Med 2015;372:1019-1030.

11. Jovin TG, Chamorro A, Cobo E, et al. Thrombectomy within 8 hours after symptom onset in ischemic stroke. $\mathrm{N}$ Engl J Med 2015;372:2296-2306.

12. Chimowitz M, Lynn M, Derdeyn C, et al. Stenting versus aggressive medical therapy for intracranial arterial stenosis. N Engl J Med 2011;365:993-1003.

13. Naylor AR. Why is the management of asymptomatic carotid stenosis so controversial? Surgeon 2015;13:34-43.

14. Marquardt I, Geraghty OC, Mehta A, Rothwell PM. Low risk of ipsilateral stroke in patients with asymptomatic carotid stenosis on best medical treatment: a prospective, population-based study. Stroke 2010;41;e11-e17.

15. den Hartog AG, Achterberg S, Moll FL, et al. Asymptomatic carotid stenosis and the risk of ischemic stroke according to 
subtype in patients with clinical manifest arterial disease. Stroke 2013;44:1002-1007.

16. Smith WS, Sung G, Starkman S, et al. Safety and efficacy of mechanical embolectomy in acute ischemic stroke: results of the MERCI trial. Stroke 2005;36:1432-1438.

17. Penumbra Pivotal Stroke Trial Investigators. The Penumbra Pivotal Stroke Trial: safety and effectiveness of a new generation of mechanical devices for clot removal in intracranial large vessel occlusive disease. Stroke 2009; 40: 2761-2768.

18. Broderick JP, Palesch YY, Demchuk AM, et al; The Interventional Management of Stroke (IMS) III Investigators. Endovascular therapy after intravenous t-PA versus t-PA alone for stroke. N Engl J Med 2013;368:893-903.

19. Kidwell CS, Jahan R, Gornbein J, et al. A trial of imaging selection and endovascular treatment for ischemic stroke. N Engl J Med 2013;368:914-923.

20. Ciccone A, Valvassori L, Nichelatti M, et al, The SYNTHESIS expansion investigators. Endovascular treatment for acute ischemic stroke. N Engl J Med 2013; 368:904-913.

21. Chimowitz M. Endovascular treatment of acute ischemic stroke-still unproven. N Engl J Med 2013;368:952-955.

22. Saver JL, Jahan R, Levy EL, et al. Solitaire flow restoration device versus the MERCI retriever in patients with acute ischemic stroke (SWIFT): a randomized, parallel group, non-inferiority trial. Lancet 2012;380:1241-1249.

23. Nogueira RG, Lutsep HL, Gupta R, et al. Trevo versus Merci retriever for thrombectomy revascularization of large vessel occlusions in acute ischemic stroke: a randomized trial (TREVO 2). Lancet 2012;380:1231-1240.
24. Campbell BC, Mitchell PJ, Kleinig TJ, et al. Endovascular therapy for ischemic stroke with perfusion-imaging selection. N Engl J Med 2015;372:1009-1018.

25. Saver JL, Goyal M, Bonafe A, et al. Stent-retriever thrombectomy after intravenous t-PA vs t-PA alone in stroke. N Engl J Med 2015;372:2285-2295.

26. Heck D. 30 day results of 227 consecutive carotid stent procedures performed in carotid stenting clinical trials. J Neurointerv Surg 2009;1:154-158.

27. Vilain KR, Magnuson EA, Li H, et al; CREST Investigators. Costs and cost-effectiveness of carotid stenting versus endarterectomy for patients at standard surgical risk: results from the Carotid Revascularization Endarterectomy versus Stenting Trial (CREST). Stroke 2012;43:2408-2416.

28. Cohen DJ, Stolker JM, Wang K, et al. Health related quality of life after carotid stenting versus carotid endarterectomy. J Am Coll Cardiol 2011;58:1577-1665.

29. Silver F, Mackey A, Clark W, et al. Safety of stenting and endarterectomy by symptomatic status in the Carotid Revascularization Endarterectomy versus Stenting Trial. Stroke 2011;42:675-680.

30. Bonati LH, Jongen LM, Haller S, et al. New ischaemic brain lesions on MRI after stenting or endarterectomy for symptomatic carotid stenosis: a sub-study of the International Carotid Stenting Study. Lancet Neurol 2010;9: 355-362.

31. Paraskevas KI, Kalmykov EL, Naylor AR. Stroke/death rates following carotid artery stenting and carotid endarterectomy in contemporary administrative dataset registries: a systematic review. Eur J Vasc Endovasc Surg 2016;51:3-12. 